\title{
The Impacts of Obstructive Sleep Apnea Severity on Brain White Matter Integrity and Cognitive Functions in Children: A Diffusion Tensor Imaging Study
}

Lin Mei, ${ }^{1, *}$ Xiaodan $\mathrm{Li}^{1}{ }^{1}{ }^{*}$ Shengcai Wang, ${ }^{1, *}$ Run $\mathrm{Si}^{2}{ }^{2}$ Tingting Ji, Zhifei $\mathrm{Xu},{ }^{3}$ Yun Peng, ${ }^{4}$ Yue Liu, ${ }^{4}$ Hongbin Li, (D)' Jie Zhang,' Yongli Guo, ${ }^{5}$ Jinghong Tian, ${ }^{6}$ Guifei Zhou, ${ }^{2}$ Huifang Huang, ${ }^{2}$ Jun Tai, ${ }^{1,7, *}$ Jiangang Liu, ${ }^{8,9}$ Xin $\mathrm{Ni}^{1}$

'Department of Otolaryngology, Head and Surgery, Beijing Children's Hospital, Capital Medical University, National Center for Children's Health, Beijing, People's Republic of China; ${ }^{2}$ School of Computer and Information Technology, Beijing jiaotong University, Beijing, People's Republic of China; ${ }^{3}$ Department of Respiration, Beijing Children's Hospital, Capital Medical University, National Center for Children's Health, Beijing, People's Republic of China;

${ }^{4}$ Department of Radiology, Beijing Children's Hospital, Capital Medical University, National Center for Children's Health, Beijing, People's Republic of China; ${ }^{5}$ Beijing Key Laboratory for Pediatric Diseases of Otolaryngology, Head and Neck Surgery, Beijing Pediatric Research Institute, Beijing Children's Hospital, Capital Medical University, National Center for Children's Health (NCCH), Beijing, People's Republic of China; ${ }^{6}$ Department of Neurorehabilitation, Beijing Children's Hospital, Capital Medical University, National Center for Children's Health, Beijing, People's Republic of China; ${ }^{7}$ Department of Otorhinolaryngology, Children's Hospital, Capital Institute of Pediatrics, Beijing, People's Republic of China; ${ }^{8}$ Beijing Advanced Innovation Center for Big Data-Based Precision Medicine, School of Engineering Medicine, Beihang University, Beijing, People's Republic of China; ${ }^{9}$ Key Laboratory of Big Data-Based Precision Medicine (Beihang University), Ministry of Industry and Information Technology of the People's Republic of China, Beijing, People's Republic of China

*These authors contributed equally to this work

Correspondence: Jiangang Liu

Beijing Advanced Innovation Center for Big

Data-Based Precision Medicine, School of

Medicine and Engineering, Beihang University,

Beijing, I00191, People's Republic of China

Email jgliu@buaa.edu.cn

Xin $\mathrm{Ni}$

Department of Otolaryngology, Beijing

Children's Hospital, Capital Medical University,

National Center for Children's Health, No. 56

Nanlishilu, Beijing, People's Republic of China

Tel +86-139-1011-1999

Email nixin@bch.com.cn
Objective: To investigate the impacts of obstructive sleep apnea (OSA) on white matter (WM) integrity and cognitive functions of pediatric patients with different levels of OSA severity.

Methods: Fifty-eight children with OSA and thirty-four healthy controls (HC) were recruited. All participants underwent diffusion tensor imaging (DTI) examination, polysomnography (PSG), and neurocognitive assessments. Patients were divided into mild OSA (MG) and moderate-severe OSA (SG) groups. WM integrity, PSG data, and neurocognitive assessment scores were compared among those groups.

Results: For apnea hypopnea index (AHI), obstructive apnea hypopnea index (OAHI), arousal index, $\mathrm{SpO}_{2}$ nadir, and attention, $\mathrm{SG}$ was worse than both $\mathrm{MG}$ and $\mathrm{HC}$ with $\mathrm{MG}$ worse than $\mathrm{HC}$. For baseline $\mathrm{SpO}_{2}$ and intelligence, SG was worse than both $\mathrm{MG}$ and $\mathrm{HC}$ with no significant difference between MG and HC. Impaired WM integrity was observed in bilateral anterior thalamic radiation, bilateral inferior fronto-occipital fasciculus, bilateral inferior longitudinal fasciculus, right superior longitudinal fasciculus, right hippocampus, left cingulate gyrus, right uncinate fasciculus, callosum forceps major, and callosum forceps minor only for SG than for HC. WM integrity was significantly correlated with OSA severity and neurocognitive assessment scores only for SG, but not for MG.

Conclusion: Decreased baseline $\mathrm{SpO}_{2}, \mathrm{WM}$ impairment, and intelligence decline were all observed only for SG, but not for MG, implying an associated relationship among decreased $\mathrm{SpO}_{2}$, WM impairment and WM impairment. Thus, for SG, additional assessments of brain damage and cognitive function decline are needed for prognostic evaluation of OSA.

Keywords: diffusion tensor imaging, obstructive sleep apnoea, children, tract-based spatial statistics, white matter

\section{Introduction}

Obstructive sleep apnea (OSA) is a common disease in children with characteristics of recurrent partial or complete upper airway obstruction. According to the guidelines published by the American Academy of Pediatrics in 2012, morbidity from OSA in children varied from $1 \%$ to $5 \% .{ }^{1}$ More and more evidence has demonstrated that long-term existence of OSA could lead to cognitive dysfunction in children. For example, Bourke et al reported that multiple cognitive and behavioral abnormalities were observed for severe sleep-disordered breathing (SDB) children. ${ }^{2}$ A recent study also indicated that OSA children exhibited lower scores in verbal skills IQ (VIQ) and full scale IQ (FIQ), along with poor 
performance in comprehension and classification test, particularly for those aged under 6 years. ${ }^{3}$ A meta-analysis of neuropsychological effects of OSA, including 1,092 patients with OSA and 899 healthy controls, indicated that the ability of OSA patients to pay attention was markedly impaired. ${ }^{4}$ However, the exact neural mechanism underlying such cognitive decline of OSA patients, particularly for OSA children, is currently unclear.

Many recent studies have found that OSA patients present with structural or functional impairment in a variety of brain regions such as pre-frontal areas, limbic lobe, insula, cingulum bundle, corpus callosum, temporal and corona radiata regions, ${ }^{5,6}$ some of which have been generally demonstrated to be involved in various cognitive processes, such as corpus callosum for the processing of memory and sustained attention, ${ }^{7}$ temporal and pre-frontal areas for the perception of emotions and cognition. ${ }^{8,9}$ In particular, some neuroimaging studies reported that some OSA patients who presented with cognitive decline (for both adults and children) also presented with brain impairment. For example, a brain morphological study of OSA showed that adults with OSA exhibited an abnormality in volume of gray matter (GM), and impairments of the hippocampus and the caudate, accompanied by reduced performance in verbal memory test. ${ }^{10}$ fMRI research showed that the inter-hemispheric effective connectivity was reduced in OSA patients who also presented with poor performance in cognitive evaluation. ${ }^{11}$ As for pediatric patients, similar to adult patients, a pediatric OSA study found reduced GM volumes in the frontal cortex, the parietal cortex, and the temporal lobes in children with OSA, and furthermore the neurocognitive assessments with Differential Ability Scales (DAS) indicated that children with OSA had lower scores than normal control children. ${ }^{12}$ Another study using magnetic resonance spectroscopy (MRS) and neuropsychological test found altered neuronal metabolite and reduced neuronal density in the hippocampus and the frontal cortex of severe OSA children who presented with significant deficits in verbal skills IQ and executive functions. ${ }^{13}$ These findings implied that there may be a close relationship between brain impairment and cognitive decline. However, the specific mechanism of this relationship is still unclear. Particularly for children, the negative impacts of OSA on both brain and cognitive development are noteworthy.

Usually, OSA in children is often accompanied by intermittent hypoxemia. It has been reported that frequent intermittent hypoxemia can lead to structural or functional brain lesions. ${ }^{14,15}$ Especially for white matter (WM), it receives a disproportionately low blood supply and rarely exhibits anastomotic branches or effective collateral circulation, and thus, it is vulnerable to damage due to recurrent hypoxemia. This may account for the findings of previous OSA studies which showed that some OSA patients presented with WM impairment. ${ }^{16-18}$ On the other hand, cognitive processing of the brain is dependent on not only the activity of brain regions, but also the connections among these regions, ${ }^{19}$ of which underlying WM connections, like functional connectivity, play an important role. ${ }^{20}$ It has been demonstrated by converging evidence that lesions in WM were related to cognitive decline. ${ }^{20,21}$ These existing findings suggest a strong correlation among intermittent hypoxemia, WM integrity impairment, and cognitive decline in OSA patients.

To test this hypothesis, the present study used diffusion tensor imaging (DTI) and cognitive assessment to explore the WM impairment and cognitive decline of children with different levels of OSA severity. DTI is sensitive for detecting WM alterations. Specifically, physical connections between brain regions at the macroscopic level could be built by simulating bundles of axonal tracts. ${ }^{22}$ Different from previous neuroimaging studies about children with OSA, the present study classified OSA children into two groups according to the severity of OSA. Then we made comparison of the outcomes of OSA, WM integrity, and cognitive functions among the non-snoring healthy controls and groups of OSA children, and explored whether they presented with similar change patterns for different levels of OSA severity.

\section{Method}

\section{Participants}

The present study was performed in compliance with the Declaration of Helsinki and approved by the Human Ethics Committees of Beijing Children's Hospital, Capital Medical University (IEC-C-028-A10-V.05 2017k-58). The enrolled children and their guardians were informed of the content and process of the present study. The informed consents were signed by respective legal guardians for children under 6 years of age, and themselves and their respective legal guardians for children over 6 years of age. Between Jan. 1, 2018 and Jan. 1, 2020, Beijing Children's Hospital recruited children aged 4-12 years with suspected OSA who had consecutive 
symptoms with habitual snoring ( $\geqq 3$ night per week). Through screening, fifty-eight right-handed children with newly diagnosed OSA, before any treatment, were selected for inclusion in the present study. Thirty-four right-handed and age-matched non-snoring children were recruited as healthy controls from the community, and were defined as $\mathrm{HC}$ group. All recruited children underwent dedicated DTI examination, full-night polysomnography (PSG), and neurocognitive assessments. According to the severity of OSA, the children were divided into two groups, namely mild (MG) and moderatesevere (SG) groups. SG included 4 moderate and 32 severe OSA children, and MG included 22 mild OSA children. The height and weight of the children were measured using stadiometers and calibrated scales, respectively. According to Chinese standards, body mass index (BMI) scores $>24 \mathrm{~kg} / \mathrm{m}^{2}$ and $>28 \mathrm{~kg} / \mathrm{m}^{2}$ are defined as overweight and obese, respectively. ${ }^{23}$

\section{Exclusion Criteria}

Children were excluded if they used psychostimulant medications or suffered from neurodevelopmental delays, dyslipidemia, congenital heart disease, diabetes mellitus, or hypertension. Children with acute or chronic illness, or a history of previous treatment for OSA were also excluded. Additionally, children with metallic implants or claustrophobia who were unable to complete MRI scanning and those whose images presented head-motion or other artefacts were excluded.

\section{Overnight Polysomnography (PSG)}

All recruited children underwent PSG monitoring (Compumedics E; Compumedics, Melbourne, Australia; or ALICE 5; Philips Respironics, Amsterdam, Netherlands) at Beijing Children's Hospital. Coffee, tea or sedative hypnotics were forbidden before overnight PSG monitoring to guarantee 7.5 hours of effective sleep. PSG parameters such as oxygen saturation $\left(\mathrm{SaO}_{2}\right)$, apnea hypopnea index (AHI), obstructive apnea hypopnea index (OAHI), total sleep time (TST), sleep efficiency (SE), and arousal index were recorded. According to the scoring manual published by the American Academy of Sleep Medicine (AASM), sleep data were calculated and evaluated by experienced pediatric PSG technicians. Children were diagnosed with OSA based on International Classification of Sleep Disorders-Third Edition (ICSD-3) diagnostic criteria of pediatric OSA published by the AASM. ${ }^{24}$ In the present study, OSA children were classified into 2 groups according to the severity of OSA, namely MG (1 events/hr $\leqq$ OAHI $<5$ events/hr) and SG (OAHI $\geqq 5$ events/hr), including moderate OSA patients ( 5 events $/ \mathrm{hr} \leqq \mathrm{OAHI}<10$ events/hr) and severe OSA patients (OAHI 10 events/hr).

\section{Neurocognitive Assessments}

The Chinese version of the Wechsler Intelligence ScaleRevised (WISC-R) for Children, provided by Xinhua Hospital, is widely used in intelligence-test-center of hospitals in China. ${ }^{25}$ The intelligence scale was used to estimate the cognitive abilities of the recruited children over 6 years of age (including 52 boys and 24 girls) by a qualified and experienced neuropsychiatrist the day after PSG examination. Meanwhile, China-Wechsler Younger Children Scale of Intelligence (C-WYCSI) was used to estimate the cognitive abilities of the recruited children under 6 years of age (including 12 boys and 4 girls). Both of these two scales of cognitive function were measured through verbal skills IQ (VIQ) and performance skills IQ (PIQ). VIQ reflects knowledge breadth, judgment and computational abilities, and PIQ reflects the abilities for practical problem-solving, spatial and visual perception. Scores for VIQ and PIQ are used to calculate a composite score as full-scale IQ (FIQ). The final FIQ, VIQ and PIQ scores were obtained by converted respective raw scores in consideration of ages and norm standardized. Higher scores indicate better neurocognitive performance.

All children also performed attention assessments by simple reaction tests that were invented for clinical application by Di Kang's biotechnological corporation. ${ }^{26}$ The main purpose of this assessment is to evaluate the child's visual and auditory attention. Correct response time and number of errors when children failed to distinguish the mismatch between figures on the screen and sound from the computer speaker are synthesized as composite score to calculate the final result of attention scores. Higher composite score indicates more severe impairment of attention.

\section{DTI Data Acquisition}

All children received DTI scanning using a 3-Tesla GE Scanner (Medical Systems Discovery MR750, USA) and an eight-channel phased-array head coil. General scanning time was approximately 12 minutes and the children under 6 years of age who were unable to complete the scan were sedated with chloral hydrate $(0.5 \mathrm{~mL} / \mathrm{kg})$. In the end, 11 children (including 5 healthy controls, 5 mild OSA and 1 severe OSA) received chloral hydrate during DTI scanning. 
Meanwhile, professional doctors accompanied children during the scanning to ensure their safety. A single-shot echoplanar imaging sequence was obtained for each whole-brain DTI SE-EPI with the following acquisition parameters: $\mathrm{b}$-value $=1000 \mathrm{~s} / \mathrm{mm}^{2}$, gradient directions $=60, \mathrm{TR} / \mathrm{TE}=$ $7000 / 62 \mathrm{~ms}, \mathrm{FOV}=256 \mathrm{~mm} \times 256 \mathrm{~mm}$, image matrix $=128$ $\times 128$, flip angle $=90^{\circ}$, slice thickness $=2 \mathrm{~mm}$ without gap, and voxel size $=2 \times 2 \times 2 \mathrm{~mm}^{3}$ (70 contiguous axial slices parallel to the anterior-posterior commissural line).

\section{Analysis of Clinical Data and Neurocognitive Assessment Scores}

Clinical data and cognitive test scores were analyzed using Statistical Package for Social Sciences (SPSS). Quantitative data were described using mean and standard deviation if normally distributed and the median and interquartile range if not. One-way analysis of variance (ANOVA) with Student-Newman-Keuls post hoc tests were used to compare among $\mathrm{MG}, \mathrm{SG}$ and $\mathrm{HC}$ if normally distributed and the Kruskal-Wallis with Steel-Dwass tests if not.

\section{Analysis of DTI Data}

Scanned DTI data were analyzed and pre-processed using FSL software (https://fsl.fmrib.ox.ac.uk/fsl/fslwiki/FSL). DTI Eigenvalues and parameter maps were generated by FMRIB's diffusion toolbox (FDT) in FSL. Voxel-wise statistical analysis was performed using tract-based spatial statistics (TBSS) methods. After inspection of data quality, the brain extraction tool (BET) was selected to segment brain tissue images with a b0. Then, DTI images in gradient coils were corrected by eddy current correction, and each voxel of diffusion images was subsequently aligned to a fractional anisotropy (FA) standard template by nonlinear registration. Finally, the diffusion tensor metrics, including FA, mean diffusivity (MD), axial diffusivity (AD), and radial diffusivity (RD), were calculated for each participant using dtifit command in FSL.

Next, neural fiber degeneration of whole-brain regions in the children with OSA was explored using TBSS. We conducted TBSS analysis among MG, SG, and HC groups. TBSS allows FA of local tract structures projected onto the virtual skeleton that uses the inherent anisotropy property to assess cerebral WM integrity, providing an aligned invariant representation of the middle portion of the tract. First, the FA image of each participant was registered to the high-resolution FMRIB58_FA image and then normalized to the $1 \times 1 \times 1 \mathrm{~mm}^{3}$ MNI 152 standard space. Then, a mean FA image was obtained by averaging the normalized FA images across all participants, and subsequently skeletonized to represent tracts common to all participants. A low FA threshold of 0.2 was set to project the normalized FA image of each participant to the mean FA skeleton to exclude GM and non-skeleton voxels. Finally, voxel-wise random permutation tests with contrasts of $\mathrm{HC}$ vs $\mathrm{MG}, \mathrm{HC}$ vs $\mathrm{SG}$, and $\mathrm{MG}$ vs $\mathrm{SG}$ were respectively implemented in FSL (5000 permutations). Statistical threshold was set at $p<0.05$ with family-wise error (FWE) corrected in all statistical maps, based on the threshold-free cluster enhancement (TFCE) statistic image. We selected an extended cluster threshold size (100 voxels) to prevent any appearance of unreliable findings. In addition, the other DTI Eigenvalues in the present study, namely $\mathrm{MD}, \mathrm{RD}$ and $\mathrm{AD}$ values, were analyzed among groups using the same method as that for FA.

\section{Analysis of Correlation}

The clusters of brain regions with significant differences among $\mathrm{MG}$, SG and $\mathrm{HC}$ were extracted using Marsbar toolbox (http://marsbar.sourceforge.net/download.html). The individual mean DTI index of each of these clusters was calculated using PANDA (Pipeline for Analyzing brain Diffusion Images).

Pearson correlation analyses were used to assess the relationship between the mean DTI Eigenvalues of each cluster and each of the neurocognitive assessment scores (ie, VIQ, PIQ, FIQ, and attention composite score) as well as the clinical severity indicator (ie, OAHI) across all children with OSA. Pearson correlation analysis was performed separately for MG and SG groups, for which the statistical threshold for significance was set at $p<0.05$.

\section{Result \\ Demographics}

All 92 children (64 males and 28 females) recruited in the present study completed brain DTI scanning, neurocognitive and PSG assessments. The HC group consisted of 11 females and 23 males, aged $8.24 \pm 2.27$ years (mean \pm $\mathrm{SD})$. The MG group consisted of 10 females and 12 males, aged $7.95 \pm 2.59$ years. The SG group consisted of 7 females and 29 males, aged $7.36 \pm 2.5$ years. Demographic results are summarized in Table 1. There were no statistically significant differences in age $(p=$ 
$0.32)$, gender $(p=0.1)$, or BMI $(p=0.53)$ among $\mathrm{HC}$, MG and $\mathrm{SG}$ groups.

\section{The Results of Analysis of Clinical Data}

The results of the comparison of PSG outcomes among HC, MG and SG groups are summarized in Table 1. There were significant differences among the groups in AHI $(p<$ $0.001)$, OAHI $(p<0.001)$, arousal index $(p<0.001)$, baseline $\mathrm{SpO}_{2}(p<0.001)$, and $\mathrm{SpO}_{2}$ nadir $(p<0.001)$. Post hoc analysis revealed two change patterns among HC, MG, and SG for these PSG outcomes. First, for AHI, OAHI, arousal index, and $\mathrm{SpO}_{2}$ nadir, $\mathrm{SG}$ was worse than both $\mathrm{MG}$ and $\mathrm{HC}(p \mathrm{~s}<0.001)$, and further, MG was worse than $\mathrm{HC}(p \mathrm{~s}<0.001$, except $p=0.016$ for $\mathrm{HC}$ vs MG of $\mathrm{SpO}_{2}$ nadir). Second, for baseline $\mathrm{SpO}_{2}$, SG was worse than both MG and $\mathrm{HC}$ (SG vs $\mathrm{HC}, p<0.001$; SG vs MG, $p=0.002$ ), whereas there was no significant difference between MG and HC ( $p=0.59)$. No differences were observed in TST $(p=0.13)$ or SE $(p=0.054)$ among these groups.

\section{The Results of Analysis of Neurocognitive Assessments}

Results of neurocognitive tests with the WISC-R and C-WYCSI were synthesized and presented in Table 1. There were significant differences among the groups in FIQ $(p=0.009)$, VIQ $(p=0.004)$, PIQ $(p=0.017)$ and attention test $(p<0.001)$.

Parallel to PSG outcomes, post hoc analysis also revealed two change patterns among these scores of neurocognitive tests. First, for attention, SG was worse than both MG and $\mathrm{HC}(p \mathrm{~s}<0.001)$, and further MG was worse than $\mathrm{HC}(p<0.001)$, consistent with the change pattern of OAHI, $\mathrm{SpO}_{2}$ nadir, and so on. Second, for FIQ, VIQ, and FIQ, SG was worse than both MG and HC $(p \mathrm{~s}<0.05)$, whereas there was no significant difference between $\mathrm{MG}$ and $\mathrm{HC}(p \mathrm{~s}>0.05)$, consistent with the change pattern of baseline $\mathrm{SpO}_{2}$.

\section{The Results of DTI Analysis}

Compared to HC, SG presented with significantly reduced FA values in 5 large clusters that extended to some brain regions such as the bilateral temporal lobes, the bilateral limbic lobe, and the bilateral sub-lobar regions, which were located in different WM tracts. These WM tracts and the brain regions with the most decreased FA in respective tracts were summarized in Table 2 .
Specifically, the WM tracts of cluster 1 included the right superior longitudinal fasciculus, the bilateral anterior thalamic radiation, the right hippocampus, the left cingulate gyrus, the right inferior fronto-occipital fasciculus, the callosum forceps major, and the callosum forceps minor. The WM tracts of cluster 2 included the right inferior longitudinal fasciculus, the right inferior fronto-occipital fasciculus, and the right uncinate fasciculus. The WM tracts of cluster 3 included the right inferior frontooccipital fasciculus and the callosum forceps major. The WM tracts of cluster 4 included the right inferior frontooccipital fasciculus, the right inferior longitudinal fasciculus, and the right anterior thalamic radiation. The WM tracts of cluster 5 included the left inferior longitudinal fasciculus, the left anterior thalamic radiation, and the left inferior fronto-occipital fasciculus. Figure 1 illustrated the fiber tracts with decreased FA values when comparing SG with HC. However, no significant difference in FA was observed when comparing SG with MG, or when comparing MG with HC. Additionally, no significant difference was observed for MD values, $\mathrm{RD}$ values, or $\mathrm{AD}$ values, among $\mathrm{HC}, \mathrm{MG}$ and $\mathrm{SG}(p \mathrm{~s}>0.05)$.

\section{The Results of Correlation Analysis}

Across all children of SG, Pearson correlation analysis was performed between the mean FA of each cluster and each of the neurocognitive assessment scores as well as the clinical severity indicator. The results were summarized in Table 3 and exhibited in Figure 2. For the children in SG, the mean FA value of cluster 2 was positively correlated with VIQ $(r=0.4, p=0.01)$. The mean FA value of cluster 3 was positively correlated with FIQ ( $r=$ $0.36, p=0.03)$ and VIQ $(r=0.38, p=0.02)$, and negatively correlated with attention scores $(r=-0.35 p=0.04)$. The mean FA value of cluster 4 was negatively correlated with OAHI ( $r=-0.39 p=0.02$ ), and positively correlated with FIQ ( $r=0.44, p=0.008)$ and VIQ $(r=0.47, p=0.004)$. The same correlation analysis was performed for $\mathrm{MG}$ as for SG. In contrast, no significant correlation was observed for MG.

\section{Discussion}

The study aimed to investigate the difference in WM integrity and cognitive function among children with different levels of OSA severity, and thereby examine the influence of OSA progression on cognitive function and WM integrity, and provide clues for the formulation of early intervention programs. At the same time, we appeal 
Table I Demographic, Sleep, and Neurocognitive Characteristics of Healthy Controls and Children with OSA

\begin{tabular}{|c|c|c|c|c|}
\hline Variables & HC $(n=34)$ & MG $(n=22)$ & SG $(n=36)$ & $p$-value \\
\hline Age (years) & $8.24 \pm 2.27$ & $7.95 \pm 2.59$ & $7.36 \pm 2.50$ & 0.32 \\
\hline Gender (F/M) & $11 / 23$ & $10 / 12$ & $7 / 29$ & 0.1 \\
\hline BMI $\left(\mathrm{kg} / \mathrm{m}^{\wedge} 2\right)$ & $19.49 \pm 6.15$ & $17.90 \pm 3.79$ & $18.99 \pm 4.84$ & 0.53 \\
\hline $\mathrm{AHI}$ (events/hr) & $0.1(0,0.425)^{* \dagger}$ & $2.4(1.6,3.55)^{\dagger}$ & $29.2(16.73,42)^{*}$ & $<0.001$ \\
\hline OAHI (events/hr) & $0^{*} \dagger$ & $1.6(1.02,2.4)^{\dagger}$ & $23.25(13.63,39.65)^{*}$ & $<0.001$ \\
\hline TST (min) & $451.58 \pm 38.67$ & $480.27 \pm 65.94$ & $463.79 \pm 52.96$ & 0.13 \\
\hline SE (\%) & $80.26 \pm 8.77$ & $84.63 \pm|0.3|$ & $85.63 \pm 9.74$ & 0.054 \\
\hline Arousal Index (events/hr) & $0(0,0.15)^{*+\dagger}$ & $1.80(1.08,2.84)^{\dagger}$ & $8.32(4.92,11.24)^{*}$ & $<0.001$ \\
\hline Baseline $\mathrm{SpO}_{2}(\%)$ & $98(97,98)^{\dagger}$ & $98(97,98)^{\dagger}$ & $97(96.25,97)^{*}$ & $<0.001$ \\
\hline $\mathrm{SpO}_{2}$ nadir (\%) & $95(93,96)^{* \dagger}$ & $93(91.75,94.25)^{\dagger}$ & $81(74.25,88.75)^{*}$ & $<0.001$ \\
\hline FIQ & $102.2 \pm 7.98^{\dagger}$ & $102.9 \pm 8.67^{\dagger}$ & $96.28 \pm 10.75^{*}$ & 0.009 \\
\hline VIQ & $101.94 \pm 6.58^{\dagger}$ & $102.23 \pm 7.34^{\dagger}$ & $96.11 \pm 9.83^{*}$ & 0.004 \\
\hline PIQ & $103.21 \pm 10.98^{\dagger}$ & $103.81 \pm 11.48^{\dagger}$ & $96.36 \pm 11.62^{*}$ & 0.017 \\
\hline Attention & $11.53 \pm 5.2^{* t}$ & $20.77 \pm 11.54^{\dagger}$ & $37.22 \pm 12.06^{*}$ & $<0.001$ \\
\hline
\end{tabular}

Notes: Values are mean \pm SD and the median and interquartile range. $P<0.05$ considered significant. *vs MG; ${ }^{\dagger}$ vs SG.

Abbreviations: MG, mild OSA group; SG, moderate-severe OSA group; HC, healthy control group; BMI, body mass index; AHI, apnea hypopnea index; OAHI, obstructive apnea hypopnea index; TST, total sleep time; SE, sleep efficiency; VIQ, verbal skills IQ; PIQ, performance skills IQ; FIQ, full scale IQ.

to parents and all of society to care for the sleep health of children through our scientific and objective evaluation of OSA cognitive functioning.

\section{Findings from the Analysis of Clinical Data and Neurocognitive Assessment \\ Scores}

The change pattern of OAHI values among $\mathrm{HC}, \mathrm{MG}$, and SG was similar to arousal index and $\mathrm{SpO}_{2}$ nadir, suggesting that the decline in sleep quality and the occurrence of anoxic events appeared even in the children with mild OSA, and further deteriorated in those with moderate or severe OSA. In parallel with these clinical findings, the children with mild OSA also presented with a significant decline in attention, which was increasingly severe from MG to SG. These findings not only suggest that the attention of children with OSA has been impaired even in the mild stage of OSA, but also implies that attention impairment may be closely associated with those two outcomes of OSA.

In contrast, the change pattern of baseline $\mathrm{SpO}_{2}$ among $\mathrm{HC}, \mathrm{MG}$, an SG was different from that of OAHI. Specifically, a significant decrease in baseline $\mathrm{SpO}_{2}$ was observed only when comparing SG with each of $\mathrm{MG}$ and $\mathrm{HC}$, but not between MG and HC. For the children in SG, frequent intermittent hypoxia could lead to significant decrease in $\mathrm{SpO}_{2}$. But for the children in $\mathrm{MG}$, limited hypoxia may only lead to a small number of times of transient decrease in $\mathrm{SpO}_{2}$. Therefore, effective $\mathrm{SpO}_{2}$ can be maintained during most sleep periods. This may be the reason why, in the present study, children in SG were associated with decreases in both $\mathrm{SpO}_{2}$ nadir and baseline $\mathrm{SpO}_{2}$, whereas children in $\mathrm{MG}$ were only associated with decrease in $\mathrm{SpO}_{2}$ nadir. VIQ, PIQ, and FIQ showed the same change patterns among $\mathrm{HC}, \mathrm{MG}$, and $\mathrm{SG}$ as that of baseline $\mathrm{SpO}_{2}$. The similarity of the change patterns among $\mathrm{HC}, \mathrm{MG}$, and $\mathrm{SG}$ for baseline $\mathrm{SpO}_{2}$ and some cognitive functions (ie, VIQ, PIQ, and FIQ) suggested that the decrease in baseline $\mathrm{SpO}_{2}$ may be closely related to the decline of these cognitive functions.

Previous studies have found decline of cognitive functions of children with OSA. ${ }^{2-4}$ The clinical and cognitive findings of the present study are consistent with those previous studies. Furthermore, the present study revealed that some of the outcomes of OSA along with some of the cognitive decline were closely related to the severity of OSA.

\section{Findings from the Analysis of DTI Data}

To examine the brain impairment in different levels of OSA severity, and the relationship between this impairment and the decline of cognitive functions, the present study compared the WM integrity among $\mathrm{HC}, \mathrm{MG}$, and SG. The results showed that no significant difference in WM integrity was observed when comparing $\mathrm{HC}$ with MG, and MG with SG. This is consistent with our previous research, ${ }^{27}$ whereas, when comparing SG with HC, 
Table 2 The Fiber Tracts with Decreased FA Values When Comparing SG with HC

\begin{tabular}{|c|c|c|c|c|c|c|c|c|}
\hline \multirow[t]{2}{*}{ Cluster No. } & \multirow[t]{2}{*}{ Voxel Size } & \multirow[t]{2}{*}{ Brain Areas } & \multirow[t]{2}{*}{ White Matter Tract } & \multicolumn{3}{|c|}{$\begin{array}{l}\text { Peak MNI } \\
\text { Coordinate }\end{array}$} & \multirow[t]{2}{*}{$t$ value } & \multirow[t]{2}{*}{$p$ value } \\
\hline & & & & $\mathbf{x}$ & $\mathbf{Y}$ & $\mathbf{z}$ & & \\
\hline \multirow[t]{8}{*}{1} & \multirow[t]{8}{*}{2403} & Left limbic lobe & Superior longitudinal fasciculus $R$ & -13 & -17 & 31 & 4.23 & 0.032 \\
\hline & & Right sub-lobar & Anterior thalamic radiation $\mathrm{R}$ & 22 & -44 & 28 & 4.10 & 0.038 \\
\hline & & Left sub-lobar & Anterior thalamic radiation $\mathrm{L}$ & -15 & -35 & 28 & 2.70 & 0.036 \\
\hline & & Right limbic lobe & Cingulum (hippocampus) $\mathrm{R}$ & 18 & -48 & 22 & 3.10 & 0.039 \\
\hline & & Left limbic lobe & Cingulum (cingulate gyrus) L & -14 & -26 & 30 & 2.50 & 0.035 \\
\hline & & Right sub-lobar & Inferior fronto-occipital fasciculus $\mathrm{R}$ & 26 & $-4 \mid$ & 27 & 2.08 & 0.040 \\
\hline & & Right sub-lobar & Callosum forceps major & 5 & -35 & 17 & 3.39 & 0.041 \\
\hline & & Right sub-lobar & Callosum forceps minor & 7 & 19 & 18 & 2.90 & 0.044 \\
\hline \multirow[t]{3}{*}{2} & \multirow[t]{3}{*}{609} & Right sub-lobar & Inferior longitudinal fasciculus R & 42 & -21 & -10 & 5.71 & 0.045 \\
\hline & & Right sub-lobar & Inferior fronto-occipital fasciculus $\mathrm{R}$ & 36 & -8 & -16 & 2.57 & 0.049 \\
\hline & & Right temporal Lobe & Uncinate fasciculus $R$ & 38 & -7 & -19 & 3.36 & 0.049 \\
\hline \multirow[t]{2}{*}{3} & \multirow[t]{2}{*}{311} & Right sub-lobar & Inferior fronto-occipital fasciculus $\mathrm{R}$ & 31 & -48 & 20 & 3.91 & 0.044 \\
\hline & & Right sub-lobar & Callosum forceps major & 32 & -56 & 15 & 2.94 & 0.045 \\
\hline \multirow[t]{3}{*}{4} & \multirow[t]{3}{*}{187} & Right sub-lobar & Inferior fronto-occipital fasciculus $\mathrm{R}$ & 33 & -22 & -4 & 4.48 & 0.048 \\
\hline & & Right sub-lobar & Inferior longitudinal fasciculus R & 28 & -23 & -3 & 2.72 & 0.049 \\
\hline & & Right sub-lobar & Anterior thalamic radiation $\mathrm{R}$ & 29 & -23 & -7 & 2.08 & 0.049 \\
\hline \multirow[t]{3}{*}{5} & \multirow[t]{3}{*}{129} & Left sub-lobar & Inferior longitudinal fasciculus L & -32 & -51 & 14 & 3.60 & 0.049 \\
\hline & & Left sub-lobar & Anterior thalamic radiation $\mathrm{L}$ & -27 & -48 & 21 & 3.46 & 0.049 \\
\hline & & Left temporal Lobe & Inferior fronto-occipital fasciculus $L$ & -29 & -47 & 18 & 3.01 & 0.049 \\
\hline
\end{tabular}

Note: Threshold $p<0.05$, FWE corrected and cluster size $>100$ voxels.

Abbreviations: L, left side; R, right side; SG, moderate-severe OSA group; HC, healthy control group; FA, fractional anisotropy.

significant FA decrease was observed in some brain regions. Additionally, for SG, the FA values of impaired brain regions in cluster 4 were negatively correlated with the OAHI value. In other words, for children with moderate and severe OSA, the more severe OSA, the more impaired the WM integrity of those brain regions. However, such correlation between the severity of OSA and the impairment of brain was not observed for children in $\mathrm{MG}$.

In agreement with the present study, previous studies of adults with OSA also reported decreased FA in some WM regions. ${ }^{16-18}$ Particularly, Macey et al compared adults with moderate and severe OSA with control participants, finding decreased FA in some WM tracts such as the left inferior longitudinal fasciculus, the left inferior fronto-occipital fasciculus, and the right superior longitudinal fasciculus, ${ }^{17}$ which are similar to those found in the present study. Thus, the findings of the present study demonstrate that, like adults with OSA, children with OSA present with impaired WM integrity in some brain regions. Furthermore, the findings of the present study suggest that, for children with OSA, the WM integrity was not impaired until the stages of moderate and severe OSA, during which the WM impairment deteriorates with the progression of OSA.

It is noteworthy that the change pattern of WM integrity measured by FA among $\mathrm{HC}, \mathrm{MG}$, and $\mathrm{SG}$ was similar to that of the cognitive functions measured by VIQ, PIQ and FIQ. Furthermore, like the severity of OSA, the severity of the impairment of some cognitive functions (eg, VIQ and FIQ) was significantly positively correlated with that of WM integrity of some brain regions only for the children with moderate and severe OSA (ie, SG), but no such correlation was significant for those with mild OSA (ie, MG). Such similarity in the change patterns of WM integrity and cognitive function modulated by OSA severity suggests that there is a possible close relationship between them. It has been reported that decreased FA values indicate axonal destruction or demyelination of WM fibers. ${ }^{22}$ Thus, impaired WM integrity of a brain region reflected by decreased FA indicates the reduced connection of WM tracts between corresponding brain regions, which can result in the decreased ability to integrate 


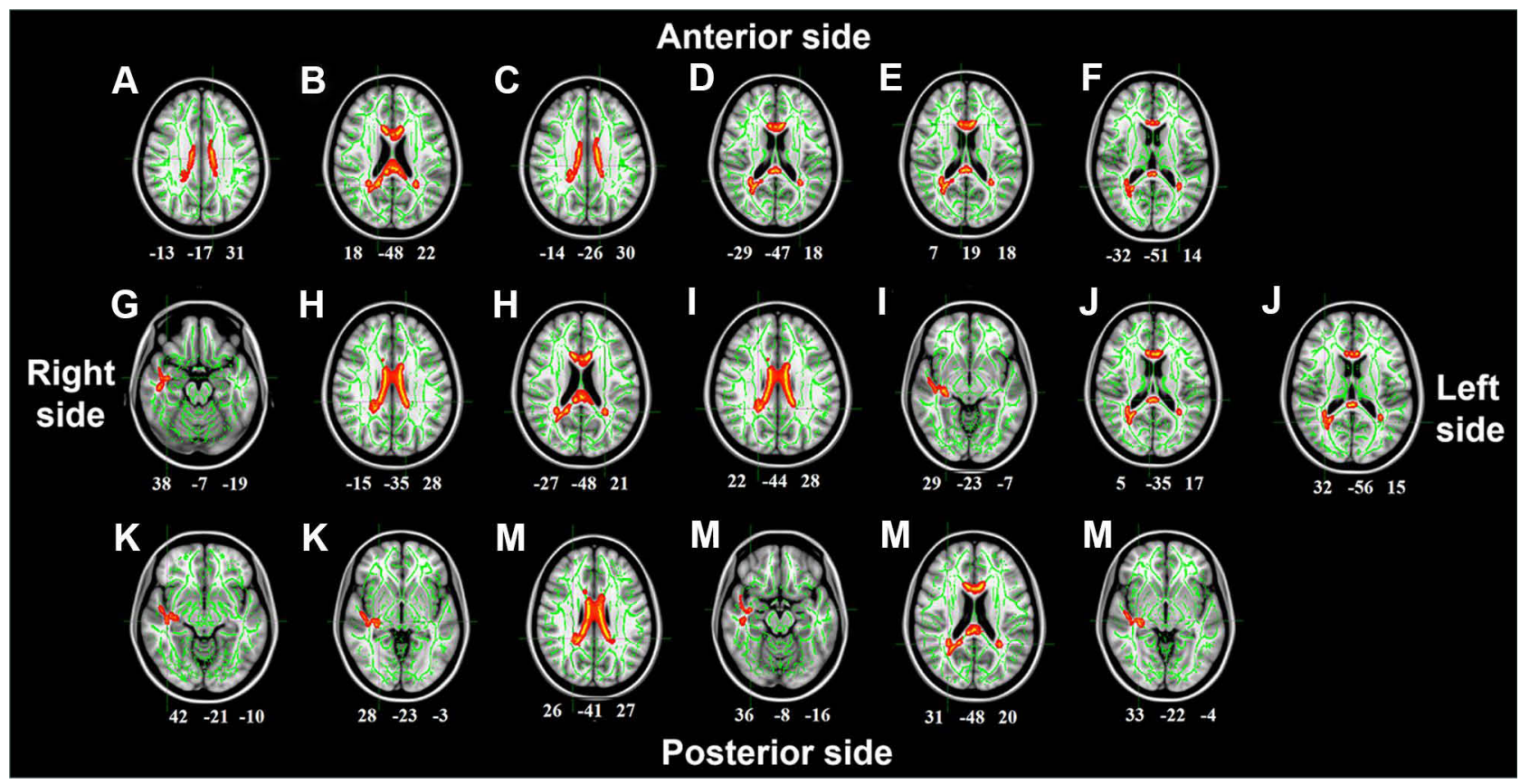

Figure I The fiber tracts with decreased fractional anisotropy (FA) values when comparing SG with HC. The green indicates the mean FA skeleton. The red-yellow illustrates the regions with decreased FA values in respective fiber tracts when comparing SG with HC. The cross mark in each sub-figure indicates the peak decrease in FA value when comparing SG with $\mathrm{HC}$ for each fiber tract of each cluster. The numbers in each sub-figure indicate the Montreal neurological institute (MNI) atlas coordinates. To allow better visualization, fiber tracts with significantly decreased FA values were thickened. (A) Superior longitudinal fasciculus R; (B) cingulum (hippocampus) R; (C) cingulum (cingulate gyrus) L; (D) inferior fronto-occipital fasciculus L; (E) forceps minor; (F) inferior longitudinal fasciculus L; (G) uncinate fasciculus R; (H) anterior thalamic radiation L; (I) anterior thalamic radiation $\mathrm{R}$; (J) forceps major; (K) inferior longitudinal fasciculus $\mathbf{R}$; (M) inferior fronto-occipital fasciculus $\mathbf{R}$. (Statistical threshold $p<0.05$, FWE corrected and cluster size > 100 voxels; images were presented in radiological convention).

Abbreviations: HC, healthy controls; SG, moderate-severe OSA group; L, left side; R, right side.

information from multiple brain regions and therefore the decline of cognitive function. ${ }^{16,20}$

In fact, some brain regions and fiber tracts that showed decreased FA in the present study have been demonstrated to be associated with various cognitive functions. For example, the functions of anterior thalamic radiation, the callosum forceps major and the callosum forceps minor are associated with autonomic and cognitive function. ${ }^{28}$ The hippocampus has been verified to play a role in memory formation, goaldirected behaviors and mood processing. ${ }^{29}$ The cingulate gyrus is made up of anterior and posterior cingulate gyrus. The anterior cingulate gyrus may be involved in cognition and emotion processing, particularly executive functions, and the posterior cingulate gyrus may be involved in

Table 3 Results of Correlation Analysis Between the Mean FA Value of Each Cluster and the Clinical Severity Indicator as Well as Neurocognitive Assessment Scores

\begin{tabular}{|c|c|c|c|c|c|c|c|c|c|c|}
\hline \multirow{2}{*}{$\begin{array}{l}\text { Brain } \\
\text { Areas }\end{array}$} & \multicolumn{2}{|c|}{ OAHI (Events/hr) } & \multicolumn{2}{|c|}{ FIQ } & \multicolumn{2}{|c|}{ VIQ } & \multicolumn{2}{|c|}{ PIQ } & \multicolumn{2}{|c|}{ Attention } \\
\hline & MG & SG & MG & SG & MG & SG & MG & SG & MG & SG \\
\hline $\begin{array}{l}\text { Cluster } \\
2\end{array}$ & $\begin{array}{l}r=-0.20 \\
(p=0.35)\end{array}$ & $\begin{array}{l}r=-0.18 \\
(p=0.28)\end{array}$ & $\begin{array}{c}r=0.24 \\
(p=0.28)\end{array}$ & $\begin{array}{c}r=0.24 \\
(p=0.15)\end{array}$ & $\begin{array}{c}r=0.28 \\
(p=0.19)\end{array}$ & $\begin{array}{c}r=0.40 \\
(p=0.01)\end{array}$ & $\begin{array}{l}r=0.10 \\
(p=0.65)\end{array}$ & $\begin{array}{c}r=0.05 \\
(p=0.75)\end{array}$ & $\begin{array}{l}r=-0.15 \\
(p=0.5 \mathrm{I})\end{array}$ & $\begin{array}{l}r=-0.27 \\
(p=0.1 I)\end{array}$ \\
\hline $\begin{array}{l}\text { Cluster } \\
3\end{array}$ & $\begin{array}{l}r=-0.21 \\
(p=0.35)\end{array}$ & $\begin{array}{l}r=-0.16 \\
(p=0.33)\end{array}$ & $\begin{array}{c}r=0.16 \\
(p=0.48)\end{array}$ & $\begin{array}{c}r=0.36 \\
(p=0.03)\end{array}$ & $\begin{array}{c}r=0.32 \\
(p=0.14)\end{array}$ & $\begin{array}{c}r=0.38 \\
(p=0.02)\end{array}$ & $\begin{array}{l}r=0.02 \\
(p=0.94)\end{array}$ & $\begin{array}{c}r=0.28 \\
(p=0.10)\end{array}$ & $\begin{array}{l}r=-0.14 \\
(p=0.54)\end{array}$ & $\begin{array}{l}r=-0.35 \\
(p=0.04)\end{array}$ \\
\hline $\begin{array}{l}\text { Cluster } \\
4\end{array}$ & $\begin{array}{l}r=-0.20 \\
(p=0.36)\end{array}$ & $\begin{array}{l}r=-0.39 \\
(p=0.02)\end{array}$ & $\begin{array}{c}r=0.02 \\
(p=0.93)\end{array}$ & $\begin{array}{c}r=0.44 \\
(p=0.008)\end{array}$ & $\begin{array}{c}r=0.13 \\
(p=0.57)\end{array}$ & $\begin{array}{c}r=0.47 \\
(p=0.004)\end{array}$ & $\begin{array}{c}r=-0.06 \\
(p=0.98)\end{array}$ & $\begin{array}{c}r=0.19 \\
(p=0.27)\end{array}$ & $\begin{array}{l}r=-0.21 \\
(p=0.34)\end{array}$ & $\begin{array}{l}r=-0.19 \\
(p=0.26)\end{array}$ \\
\hline
\end{tabular}

Note: $p<0.05$ considered statistically significant and presented in bold.

Abbreviations: FA, fractional anisotropy; MG, mild OSA group; SG, moderate-severe OSA group; OAHI, obstructive apnea hypopnea index; VIQ, verbal skills IQ; PIQ, performance skills IQ; FIQ, full scale IQ; cluster, brain regions with decreased FA values for SG compared to HC; HC, healthy control group. 

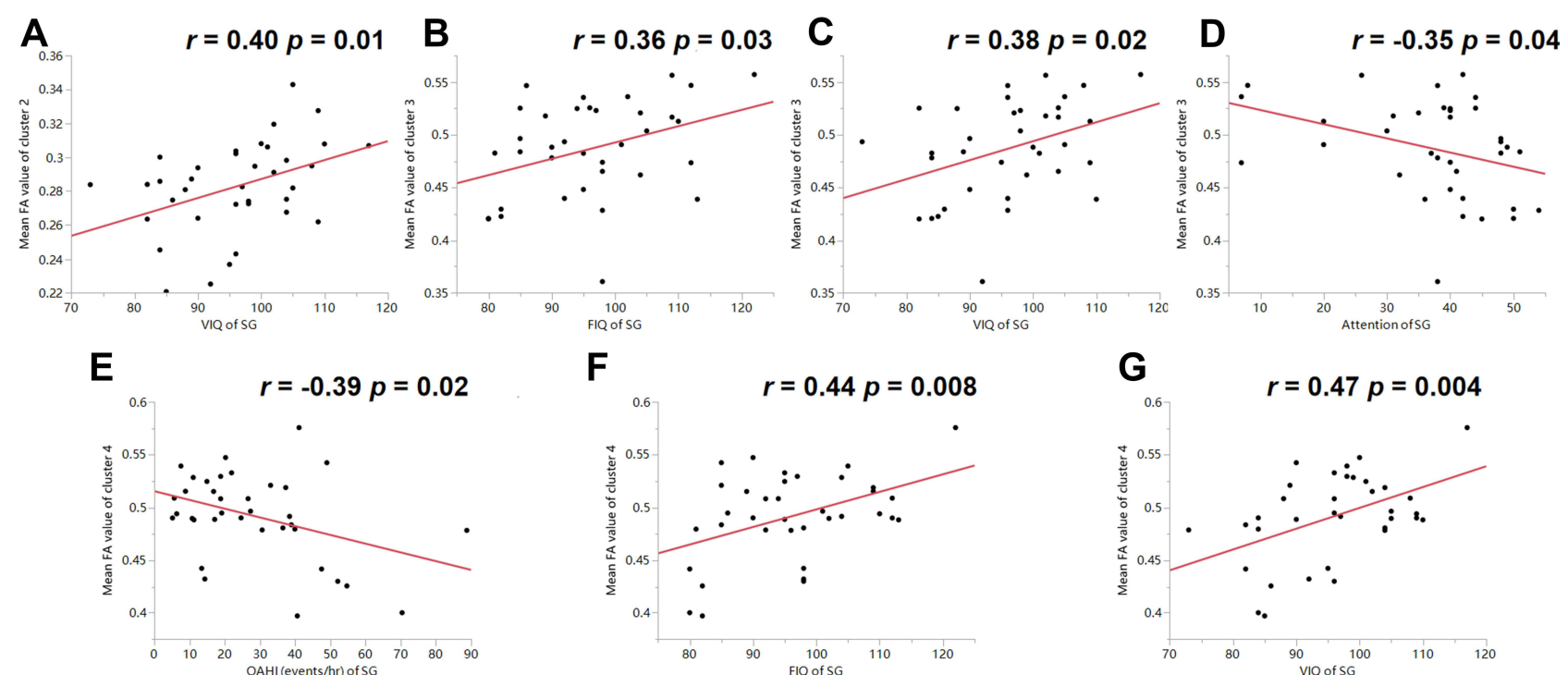

$\mathbf{F}$
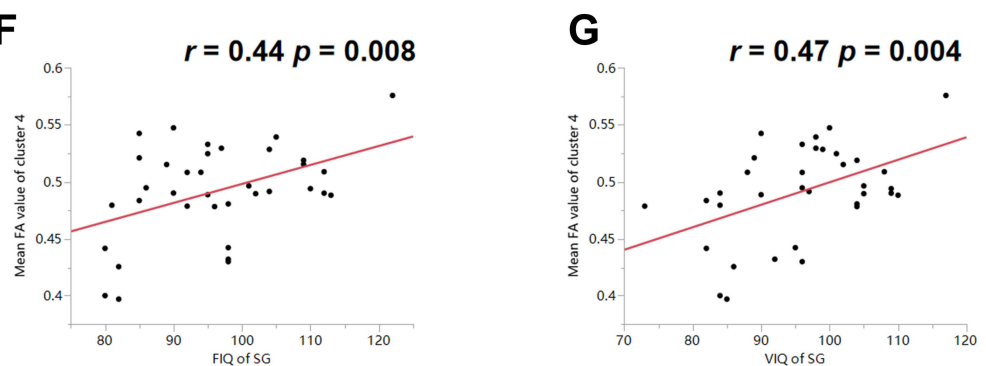

Figure 2 Correlation analysis between the mean FA value of cluster $2-4$ and the clinical severity indicator as well as neurocognitive assessment scores. (The WM tracts of cluster 2 included the right inferior longitudinal fasciculus, the right inferior fronto-occipital fasciculus, and the right uncinate fasciculus; the WM tracts of cluster 3 included the right inferior fronto-occipital fasciculus and the callosum forceps major; the WM tracts of cluster 4 included the right inferior fronto-occipital fasciculus, the right inferior longitudinal fasciculus, and the right anterior thalamic radiation). (A) Positive correlation between the mean FA value of cluster 2 and VIQ of SG; (B) positive correlation between the mean FA value of cluster 3 and FIQ of SG; (C) positive correlation between the mean FA value of cluster 3 and VIQ of SG; (D) negative correlation between the mean FA value of cluster 3 and attention results of SG; (E) negative correlation between the mean FA value of cluster 4 and OAHI of SG; (F) positive correlation between the mean FA value of cluster 4 and FIQ of SG; (G) positive correlation between the mean FA value of cluster 4 and VIQ of SG. Higher neurocognitive assessment scores (VIQ and FIQ) indicate better neurocognitive performance and higher composite attention scores indicate more severe impairment of attention.

Abbreviations: SG, moderate-severe OSA group; HC, healthy control group; OAHI, obstructive apnea hypopnea index; VIQ, verbal skills IQ; FIQ, full scale IQ; cluster, brain regions with decreased FA values for SG compared to $H C$.

memory retrieval and evaluation of information. ${ }^{30}$ The inferior fronto-occipital fasciculus connects the occipitotemporal areas to the frontal lobe, the alteration of which is related to the decline of many complex cognitive functions, such as episodic memory, social cognition, attention, and multitasking and subcomponent analysis. ${ }^{31}$ The inferior longitudinal fasciculus that links the anterior temporal lobe with the extrastriate cortex of the occipital lobe has been found to be related to autobiographical memory. ${ }^{32}$ Additionally, a recent study reported that, after 12-month continuous positive airway pressure (CPAP) treatment, the impairment of WM integrity and the decline of cognitive function of adults with OSA were both improved. ${ }^{33}$ This evidence along with the findings of the present study suggest that the impairment of WM integrity modulated by OSA severity may cause the decline of cognitive functioning in children.

Previous studies about OSA have consistently reported that OSA patients presented with some cognitive decline accompanied with brain impairments in some brain regions. ${ }^{6,11,18}$ As the extension of these previous studies, to the best of our knowledge, the present study is the first to reveal the common change patterns of the outcome of OSA (ie, baseline $\mathrm{SpO}_{2}$ ), cognitive functions and WM integrity of brain regions among pediatric groups with different levels of OSA severity, therefore implying a kind of close relationship among the severity of OSA, the impairment of brain WM integrity, and then the decline of cognitive functioning.

\section{Treatment of Children with OSA}

Fortunately, for children with OSA, the success rate of therapy, including medication (ie, intranasal corticosteroids, anti-inflammatory and leukotriene antagonist) and surgery (ie, adenoid tonsillectomy), is as high as $81 \% .^{34}$ The success, however, was defined as an amelioration of hypoxia, and it is not clear whether improved hypoxia can reverse the WM impairment and the cognitive decline that occur in moderate and severe OSA. A recent study presented that adenoid tonsillectomy could improve daytime behavior, sleep apnea symptoms, subjective sleepiness, and quality of life, but there was limited improvement in cognitive tests for attention or executive function. ${ }^{35}$ Thus, a comprehensive prognostic evaluation of pediatric OSA is needed, which includes not only the assessment of clinical symptoms of OSA, but also of brain damage and cognitive decline. 
Another important issue in pediatric OSA is whether children with mild OSA should be treated. This question is complex and difficult to deal with, because it is necessary to not only consider the clinical features and physiological states of the children with OSA, but also establish a precise balance between the potential positive and negative influence of treatment on pediatric patients. The present study revealed the relationship among the decrease in some traditional clinical features of OSA, the impairment of brain WM integrity, and the decline of cognitive function in children with different levels of OSA severity. These findings may provide supplemental information about the outcomes of pediatric OSA for diagnostic criteria, on which the decision whether or how children with mild OSA are treated, can be based.

\section{Limitations}

There are still some limitations worth considering, even though the current research has made useful observations. The insufficient sample size cannot be neglected. Thus, the inferences made in this cross-sectional study need to be further confirmed in a larger sample size, cohort study to verify WM damage and cognitive decline in OSA children. Additionally, post-treatment follow-up studies of children with OSA should be conducted to examine the amelioration of WM impairment and cognitive decline.

\section{Conclusion}

The present study explored the difference in WM integrity and cognitive function for children with different levels of OSA severity. In the present study, the children with mild OSA did not present with impairments of WM integrity or decline in intelligence. In contrast, impairments of both WM integrity of some brain regions and cognitive functioning were observed in children with moderate and severe OSA. Such change pattern was similar to that of baseline $\mathrm{SpO}_{2}$ among children with different levels of OSA severity, suggesting an associated relationship among decreased $\mathrm{SpO}_{2}$, the impairment of brain WM integrity, and the decline of cognitive functioning. The findings of the present study also provide some clues regarding the comprehensive reassessment of OSA symptoms, and the formulation of early intervention programs.

\section{Acknowledgments}

This work was supported by National Natural Science Foundation of China (grant number 81970900, 61673051); Capital Funds for Health Improvement and Research (grant number 2018-1-2091); Pediatric Medical
Coordinated Development Center of Beijing Municipal Administration (grant number XTZD20180101); Beijing Natural Science Foundation (grant number 7194262); Beijing Hospitals Authority Ascent Plan (grant number DFL20191201); and Project Beijing Advanced Innovation Center for Big Data-Based Precision Medicine, Beihang University \& Capital Medical University (grant number BHME-201911).

\section{Disclosure}

The authors report no conflicts of interest in this work.

\section{References}

1. Marcus CL, Brooks LJ, Draper KA, et al. Diagnosis and management of childhood obstructive sleep apnea syndrome. Pediatrics. 2012;130 (3):e714-755. doi:10.1542/peds.2012-1672

2. Bourke RS, Anderson V, Yang JS, et al. Neurobehavioral function is impaired in children with all severities of sleep disordered breathing. Sleep Med. 2011;12(3):222-229. doi:10.1016/j.sleep.2010.08.011

3. Zhao J, Han S, Zhang J, et al. Association between mild or moderate obstructive sleep apnea-hypopnea syndrome and cognitive dysfunction in children. Sleep Med. 2018;50:132-136. doi:10.1016/j. sleep.2018.04.009

4. Beebe DW, Groesz L, Wells C, Nichols A, McGee K. The neuropsychological effects of obstructive sleep apnea: a meta-analysis of norm-referenced and case-controlled data. Sleep. 2003;26 (3):298-307. doi:10.1093/sleep/26.3.298

5. Kumar R, Chavez AS, Macey PM, Woo MA, Yan-Go FL, Harper RM. Altered global and regional brain mean diffusivity in patients with obstructive sleep apnea. J Neurosci Res. 2012;90 (10):2043-2052. doi:10.1002/jnr.23083

6. Chen LT, Fan XL, Li HJ, et al. Aberrant brain functional connectome in patients with obstructive sleep apnea. Neuropsychiatr Dis Treat. 2018;14:1059-1070. doi:10.2147/NDT.S161085

7. Zhang B, Zhu DM, Zhao W, et al. Selective microstructural integrity impairments of the anterior corpus callosum are associated with cognitive deficits in obstructive sleep apnea. Brain Behav. 2019;9 (12): $\mathrm{e} 01482$. doi:10.1002/brb3.1482

8. Chaudhary U, Hall M, DeCerce J, Rey G, Godavarty A. Frontal activation and connectivity using near-infrared spectroscopy: verbal fluency language study. Brain Res Bull. 2011;84(3):197-205. doi:10.1016/j.brainresbull.2011.01.002

9. Nakatani H, Muto S, Nonaka Y, Nakai T, Fujimura T, Okanoya K. Respect and admiration differentially activate the anterior temporal lobe. Neurosci Res. 2019;144:40-47. doi:10.1016/j. neures.2018.09.003

10. Torelli F, Moscufo N, Garreffa G, et al. Cognitive profile and brain morphological changes in obstructive sleep apnea. Neuroimage. 2011;54(2):787-793. doi:10.1016/j.neuroimage.2010.09.065

11. Canessa N, Castronovo V, Cappa SF, et al. Sleep apnea: altered brain connectivity underlying a working-memory challenge. Neuroimage Clin. 2018;19:56-65. doi:10.1016/j.nicl.2018.03.036

12. Philby MF, Macey PM, Ma RA, Kumar R, Gozal D, KheirandishGozal L. Reduced Regional Grey Matter Volumes in Pediatric Obstructive Sleep Apnea. Sci Rep. 2017;7:44566. doi:10.1038/ srep44566

13. Halbower AC, Degaonkar M, Barker PB, et al. Childhood obstructive sleep apnea associates with neuropsychological deficits and neuronal brain injury. PLoS Med. 2006;3(8):e301. doi:10.1371/journal. pmed.0030301 
14. Xu W, Chi L, Row BW, et al. Increased oxidative stress is associated with chronic intermittent hypoxia-mediated brain cortical neuronal cell apoptosis in a mouse model of sleep apnea. Neuroscience. 2004;126(2):313-323. doi:10.1016/j.neuroscience.2004.03.055

15. Li RC, Row BW, Kheirandish L, et al. Nitric oxide synthase and intermittent hypoxia-induced spatial learning deficits in the rat. Neurobiol Dis. 2004;17(1):44-53. doi:10.1016/j.nbd.2004.05.006

16. Lee $\mathrm{MH}$, Yun $\mathrm{CH}$, Min A, et al. Altered structural brain network resulting from white matter injury in obstructive sleep apnea. Sleep. 2019;42(9):zsz120. doi:10.1093/sleep/zsz120

17. Macey PM, Kumar R, Woo MA, Valladares EM, Yan-Go FL, Harper RM. Brain structural changes in obstructive sleep apnea. Sleep. 2008;31(7):967-977.

18. Rostampour M, Noori K, Heidari M, et al. White matter alterations in patients with obstructive sleep apnea: a systematic review of diffusion MRI studies. Sleep Med. 2020;75:236-245. doi:10.1016/j. sleep.2020.06.024

19. Friston K. A theory of cortical responses. Philos Trans $R$ Soc Lond B Biol Sci. 2005;360(1456):815-836. doi:10.1098/rstb.2005.1622

20. Filley CM, Fields RD. White matter and cognition: making the connection. J Neurophysiol. 2016;116(5):2093-2104. doi:10.1152/ jn.00221.2016

21. Alfaro FJ, Gavrieli A, Saade-Lemus P, Lioutas VA, Upadhyay J, Novak V. White matter microstructure and cognitive decline in metabolic syndrome: a review of diffusion tensor imaging. Metabolism. 2018;78:52-68. doi:10.1016/j.metabol.2017.08.009

22. Le Bihan D, Mangin JF, Poupon C, et al. Diffusion tensor imaging: concepts and applications. J Magn Reson Imaging. 2001;13 (4):534-546. doi:10.1002/jmri.1076

23. Zong Y, Xie R, Deng N, et al. Secular trends in overweight and obesity among urban children and adolescents, 2003-2012: a serial cross-sectional study in Guangzhou, China. Sci Rep. 2017;7 (1):12042. doi:10.1038/s41598-017-12094-z

24. Sateia MJ. International classification of sleep disorders-third edition: highlights and modifications. Chest. 2014;146(5):1387-1394. doi:10.1378/chest.14-0970
25. Liu J, Lynn R. An Increase of Intelligence in China 1986-2012. Intelligence. 2013;41(5):479-481. doi:10.1016/j.intell.2013.06.017

26. Michael B, Steinborn HC. Methodology of Performance Scoring in the d2 Sustained-Attention Test: cumulative-Reliability Functions and Practical Guidelines. Psychol Assess. 2018;30(3):339-357. doi:10.1037/pas0000482

27. Mei L, Xiaodan L, Zhou G, et al. Effects of obstructive sleep apnoea severity on neurocognitive and brain white matter alterations in children according to sex: a tract-based spatial statistics study. Sleep Med. 2021;06(82):134-143. doi:10.1016/j.sleep.2020.08.026

28. Wang Z, Bai L, Liu Q, et al. Corpus callosum integrity loss predicts cognitive impairment in Leukoaraiosis. Ann Clin Transl Neurol. 2020;7(12):2409-2420. doi:10.1002/acn3.51231

29. Bird CM, Burgess N. The hippocampus and memory: insights from spatial processing. Nat Rev Neurosci. 2008;9(3):182-194. doi:10.1038/nrn2335

30. Bush G, Luu P, Posner MI. Cognitive and emotional influences in anterior cingulate cortex. Trends Cogn Sci. 2000;4(6):215-222. doi:10.1016/S1364-6613(00)01483-2

31. Wu Y, Sun D, Wang Y, Wang Y. Subcomponents and Connectivity of the Inferior Fronto-Occipital Fasciculus Revealed by Diffusion Spectrum Imaging Fiber Tracking. Front Neuroanat. 2016;10 (44):88. doi:10.3389/fnana.2016.00088

32. Hodgetts CJ, Postans M, Warne N, Varnava A, Lawrence AD, Graham KS. Distinct contributions of the fornix and inferior longitudinal fasciculus to episodic and semantic autobiographical memory. Cortex. 2017;94:1-14. doi:10.1016/j.cortex.2017.05.010

33. Castronovo V, Scifo P, Castellano A, et al. White matter integrity in obstructive sleep apnea before and after treatment. Sleep. 2014;37 (9):1465-1475. doi:10.5665/sleep.3994

34. Ehsan Z, Ishman SL. Pediatric Obstructive Sleep Apnea. Otolaryngol Clin North Am. 2016;49(6):1449-1464. doi:10.1016/j.otc.2016.07.001

35. Taylor HG, Bowen SR, Beebe DW, et al. Cognitive Effects of Adenotonsillectomy for Obstructive Sleep Apnea. Pediatrics. 2016;138(2):e20154458. doi:10.1542/peds.2015-4458

\section{Publish your work in this journal}

Nature and Science of Sleep is an international, peer-reviewed, open access journal covering all aspects of sleep science and sleep medicine, including the neurophysiology and functions of sleep, the genetics of sleep, sleep and society, biological rhythms, dreaming, sleep disorders and therapy, and strategies to optimize healthy sleep.
The manuscript management system is completely online and includes a very quick and fair peer-review system, which is all easy to use. Visit http://www.dovepress.com/testimonials.php to read real quotes from published authors. 